\title{
The Application and Advantages of Dynamic Illustration in Illustration Design
}

\author{
Wenquan Zhou ${ }^{1, a^{*}}$ and Dan $\mathrm{Xu}{ }^{2, \mathrm{~b}}$ \\ ${ }^{1}$ City College of WUST, Wuhan, China 430083 \\ 2 City College of WUST, Wuhan, China 430083 \\ A648874962@qq.com, b15596470@qq.com
}

\section{Keywords: Illustration; Dynamic Illustration; Information Transfer; Visual Communication}

\begin{abstract}
With the development of information technology, digital technology, the dramatic changes in people's needs, illustration is not exceptional also dynamic state illustration is developed on the basis of the traditional static illustration, and in recent years, gradually developed into people's cultural life, a popular art form, it in new visual language in modern design occupy a place in the article expounds the dynamic illustration on the illustration of the UI design ebook and the application of the logo design and advantages, dynamic illustration to make this form with a broader understanding and development.
\end{abstract}

\section{Introduction}

Illustration design development to this day, to a certain extent has to get rid of the original concept, more and more illustration design is out of the scope for the application of text explanation and tend to be more independent, especially in the moment the map reading age, illustration is widely used in every aspect of our life with two digital technology popularization, have also become highly digital illustration creation way, CG technology namely (computer graphics) has become the mainstream in the illustration drawing method, a new technology of artificial illustration design has a long history of arts is not only a technical innovation, from but make illustration design connotation and denotation expanded two particularly close In recent years, a new kind of dynamic illustration began to appear in people's vision, changing the existing expression paradigm of traditional illustration. It can be said that dynamic illustration is a new exploration in the field of painting image and digital technology.

\section{Current situation and problems}

In the Internet age, illustration works can spread with the fastest speed, realize socialization and popularization of the two network media so that the illustrator's personal expression and creativity extend to the audience, the network platform provides an artist with a fusion and collision of unlimited creative environment, and constantly promote new discoveries and new attempts in illustration art. Second, what we need to see is that contemporary illustrations have a strong timeliness and popularity, often sensitive to the impact of various cultural factors of the times, and grasp the cultural hot spot, become a pioneering art style and visual expression of ideological two plus today's illustrations are no longer limited to the use of paper media. Network and mobile media terminals have become the new front of illustration design applications, such as electronic magazines, outdoor advertising, product instructions, mobile phone application interface can see the shadow of illustration design works, new technical means and new media for the birth of dynamic illustration this new art form has inestimable work Dynamic illustration makes illustration, which has a long history, bring new vitality and become one of the most pioneering forms of expression in the era of visual communication.

The reading of characters requires the audience to obtain information through deep thinking and to appreciate its inner meaning through the way of concentration, and then comprehend the deep connotation conveyed by the author. The visual image breaks the distance between the words and the real world, dispels the indirectness, abstractness and taste of the expression of the characters, and disseminates the past. 


\section{The application and advantages of dynamic illustration}

The characteristic of dynamic illustration is that the basic form of the picture must be illustration, with painting as the basis of artistic expression, and not only a dynamic image, and dynamic illustration often only provides an image to the audience placed in the environment, intended to create a situation, no plot, no lens changes, or even No. The dynamic part, with its roles, is just another dimension in which the creator's intentions are presented at a deeper level.

In today's rapid development of computer technology, the new art form is the necessity of social progress Now, the design of dynamic has been integrating many art forms, has been shown to influence in the field of visual design, the present people's reading object Reading and reading psychology and so on great changes have taken place in looking to the future development of dynamic illustration, are faced with both opportunities and there are many challenges.

Dynamic illustration and LOGO design. For a long time, the integration of brand and illustration design is developing very rapidly, but also get people's support and recognition. In the display of traditional logo design, static graphics has occupied people's attention for a long time. With the emergence of dynamic illustration, some enterprises have adopted the combination of dynamic illustration and logo design. This combination of dynamic illustration and logo provides a new choice for designers and audiences, and helps to show the brand concept and media communication.

Logo plays a very high role in the visual image of an enterprise. People's most intuitive understanding of a brand is the logo of the enterprise. First of all, through the dynamic interpretation of the logo, it can produce a strong visual impact and attract people's attention. Secondly, the logo itself can bear a certain corporate culture, while the variable characteristics of dynamic logo graphics can integrate the internal spirit of the enterprise more completely, so as to convey the corporate culture to the outside world more intuitively and conveniently. Finally, it is more conducive for enterprises to present themselves in today's increasingly prosperous mobile media, so as to gain broader publicity space, which is conducive to the development of enterprises

Dynamic illustration and UI design. UI design is the abbreviation of User Interface. It mainly refers to the overall design of human-computer interaction, operation logic donation and beautiful interface of software. UI design is a new design field with the development of the Internet. It requires a high degree of humanization. Because UI design is directly related to user experience, user comfort is directly related to the survival of the software. The humanized design of a software involves every detail related to user use. For example, many applications involve waiting for a drop-down refresh, or loading blanks for pictures, videos, etc. Dynamic illustration can be applied to them, which can greatly improve user comfort.

First of all, for the mass illustration design how to after moving to the new forms of illustration work between the audience and resonance, the resonance from the common between artist and audience aesthetic orientation and tacit understanding, dynamic illustration of creators need keenly from goods and information in the public aesthetic standard and to feel the era pulse Second, how can you keep traditional illustration meaningful in our dynamic images of deep aesthetic artistic conception, to meet the public pursuit of novel emotional appeal at the same time, still can let the audience in his own association and thinking, even in the abstract thinking and deductive reasoning, rather than the creator for visual trend blindly cater to or indulge in the fun of narcissistic, all these problems need to create dynamic illustration artists from the boldly in the creation and practice analysis, rational solution for dynamic illustration of this new type of illustrations, its artistic value judgment is needed is a diversified and fair Objective voice needs to be supported by self established discipline theory to guide practitioners to develop in a positive and healthy direction.

The innovation of illustration. The interaction provided by technological innovation is full of new possibilities for artistic creation. Painting technology changes the way of painting, and the way of painting has an impact on the form of painting. Digital tools change the behavior of a single "painting" into "painting" and "synthesis". The process is reversible and infinite. Dynamic illustration generates a dazzling image language that breaks the visual conventions, changes the limitations of the original two-dimensional plane, improves the performance of the field of 
environmental space and visual features show that illustration has a long history of expression and compatibility, which greatly meets the aesthetic needs of the audience. For example, the dynamic effect of many dynamic illustrations is an infinite circle of closed-loop effect, its interesting greatly increased, so that the audience has been unable to get a new aesthetic experience. As a widely used art communication style in the visual communication era, illustration design is always influenced by various elements: from the change of the art style of the times, the promotion of new technology conditions to the change of the public aesthetic, all of them bring the richness of the content and the form of presentation to the illustration design.

Designers can use dynamic visual elements for bold innovation and practice, the visual elements are split and reconstructed to create more value space. Dynamic elements for illustration design intermediary is not only a kind of reference on the surface, but also a kind of inner spiritual experience of visual art. In addition to simple dynamic deduction of pictures and texts, dynamic illustration gives new meaning to illustration design, including the display of object movement track, the use of role action, the use of dynamic narrative mode, and the creation of picture atmosphere. From the expression of conception and cultural connotation, the work is more stylistic and enjoyable. It even shows a certain culture.

Static illustration and dynamic illustration. Since the advent of paper making and printing, due to the limitations of printing technology and computer technology, the spread of traditional illustrations is basically based on books, newspapers and other paper media. Traditional illustration through long-term improvement and development of the deep foundation of science and technology and the continuous progress of dynamic illustration on this basis to show a broader play space and show new features. First of all, dynamic illustration provides people with a new choice, different content determines the choice of illustration of different forms of expression; second, compared with traditional illustration, dynamic illustration for the performance of the scene atmosphere more diverse, so more superior; finally, the contrast between the dynamic and static visual impact, can bring viewers Unexpected artistic effects.

Dynamic illustration not only increases the fun of reading, but also gives readers a more intuitive and vivid impression; in narrative children's books, the advantages of dynamic illustration are more obvious, an illustration with simple words can achieve the purpose. Dynamic illustration also has great application value in all aspects of science and technology, for example, in medical books, some knowledge of anatomy can be demonstrated through dynamic illustration process, more intuitive and convenient. Compared with video, dynamic illustration is more efficient, does not need to complete a complete animation video, greatly saving production costs. Illustrations with text and video will be viewed more than 10000 to avoid readers' omission for details. Therefore, dynamic illustration is more convenient than traditional static illustration and video.

\section{Conclusion}

Technological innovation provide many possibilities for the development of art, dynamic illustration is resulting from development to today, the emergence of the network and various emerging mobile media to artist designer provides a wider platform, enabling them to firmly grasp the pulse of The Times and cultural trends, and on this basis, the more free to express ideas at the same time, various emerging mobile media for the audience to expand more choice, outside of the rich material life greatly meet the needs of the people's spiritual life, make life more interesting This kind of dynamic illustration between illustration and animation combines the advantages of both and has a more convenient communication space and a broader application field. It makes illustration, an ancient art form, radiate new vitality and develop into a pioneer expression form of contemporary visual communication.

\section{References}

[1] Q. Xu: Art Education Research, Vol. 2 (2018), p.61.

[2] Lawrence Zeegen: The Fundamentals of Illustration (Dalian University of Technology Press, 
China 2014)

[3] C.F. Wang, S.C. Xu and H.H Guan : Appreciation, Vol. 17 (2018), p.184.

[4] R. Huang: Beauty \& Times, Vol. 3 (2018) , p.120.

[5] L.Y. Zhu: Leading research on creative thinking in commercial illustration design (MS., Harbin University of Science and Technology, China 2017), p.21.

[6] Y. Liu: Journal of Donghua University(Social Science), Vol. 3 (2014), p.180.

[7] L. Ren: A Study on the Aesthetic Characteristics of Digital-Technology Cutting-edge Illustration (MS., Academi Degrees and Graduate Education of Liaocheng University, China 2017), p.14.

[8] Q. Hao: Design in Mobile H5 Advertisement (MS., Hubei Institute of Fine Arts , China 2017), p.7.

[9] W.J. Zhang: The application of integrated materials as form language in illustration design (MS., Beijin University of Technology, China 2016), p.19.

[10] Jon Krasner: Motion graphic design applied history and aesthetics (Posts \& Telecom Press, China 2016) 